\title{
NEURONAL CONSTITUTIVE AND INDUCIBLE NITRIC OXIDE SYNTHASE EXPRESSION IN THE BRAINS OF Listeria monocytogenes-INFECTED CATTLE
}

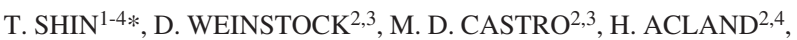 \\ M. WALTER ${ }^{2,4}$, H. KIM ${ }^{2,4}$, M. AHN ${ }^{1}$, H. G. PURCHASE ${ }^{2,4}$ \\ ${ }^{1}$ Department of Veterinary Medicine, Brain Korea 21, Institute for Life Science, Cheju National University, \\ Cheju 690-756, Republic of Korea; ${ }^{2}$ Pennsylvania Animal Diagnostic Laboratory System; \\ ${ }^{3}$ Center for Veterinary Diagnostics and Investigation, Pennsylvania State University, University Park, PA; and \\ ${ }^{4}$ Pennsylvania Veterinary Laboratory, Harrisburg, PA, U.S.A.
}

\section{Received September 4, 2000 \\ Accepted February 7, 2001}

\section{Abstract}

Taekyun Shin, D. Weinstock, M. D. Castro, H. Acland, M. Walter, Hyun Young Kim, Meejung Ahn, H. G. Purchase: Neuronal Constitutive and Inducible Nitric Oxide Synthase Expression in the Brains of Listeria monocytogenes-Infected Cattle. Acta Vet. Brno 2001, 70: 43-47.

Nitric oxide (NO) is important for intracellular signaling and neurotransmission. Nitric oxide synthase (NOS) is involved in central nervous system (CNS) disorders through excess generation of NO. In this study, we examined the expression of both constitutive and inducible nitric oxide synthase (NOS) in Listeria monocytogenes-infected brains of cattle by immunohistochemistry.

Microabscesses, focal gliosis, and perivascular cuffing characterized the histopathological changes in the brain in listeriosis. L. monocytogenes antigen was present in the microabscesses and perivascular cuffs. Mononuclear phagocytes and neutrophils were the most numerous inflammatory cells in the affected areas of the brainstem. In natural listeriosis, brain cells, including neurons and some neuroglial cells, expressed both nNOS and iNOS. The immunoreactivity of iNOS was more intense than that of the control. In some lesions, inflammatory cells (mainly macrophages) expressed nNOS and iNOS in the microabscesses and perivascular cuffs, while inflammatory neutrophils in the same lesion were not stained with iNOS.

These findings suggest that normal bovine brain cells, including neurons and neuroglia, constitutively express iNOS and nNOS, and that there is increased expression of these molecules in L. monocytogenes infections. Furthermore, inflammatory cells, including macrophages, expressing both nNOS and iNOS may play an important role in the pathogenesis of bacterial meningoencephalitis in cattle.

Listeria monocytogenes, encephalitis, nitric oxide synthase

Nitric oxide (NO) is a readily diffusible, apolar gas, synthesized from L-arginine by nitric oxide synthase (NOS) (Murphy et al. 1993). The enzyme responsible for NO formation exists in three forms: two constitutive forms, neuronal NOS (nNOS) and endothelial NOS (eNOS), and inducible NOS (iNOS). In the brain, the constitutive NOSs are most important in the initial generation of NO in the central nervous system (CNS). NO is important for intracellular signaling and neurotransmission (Moncada et al. 1991; Bredt and Snyder 1992). Besides its expected physiological role, NO is likely involved in CNS disorders, including experimental autoimmune encephalomyelitis (Van Dam et al. 1995; Cross et al. 1997), bacterial meningoencephalitis (Jungi et al. 1997), and viral encephalitis (Hooper et al. 1995; V an Dam et al. 1995; Iwahashi et al. 1999).

Listeriosis is a complementary model of an intracytoplasmic organelle infection in the central nervous system (CNS) of animals (Johns on et al. 1995) and humans (Cooper and Walker 1998). Animals affected by listeriosis show circling behavior followed, in most

Address for correspondence:

Takeyun Shin, DVM, PhD

Department of Veterinary Medicine, College of Agriculture,

Cheju 690-756, Republic of Korea
Tel: +82-64-754-3363

Fax: +82-64-756-3354;

http://www vfu.cz/acta-vet/actavet.htm 
cases, by death (Otter and Blackmore 1989; Dramsi et al. 1998). Lesions in the brain may or may not contain L. monocytogenes antigens, but characteristically consist of inflammatory cells, including neutrophils, macrophages, and some lymphocytes, suggesting that acute and chronic inflammatory cells play an important role in the brain tissue injury (Krueger et al. 1995; Jungi et al. 1997). These cell types are also associated with the secretion of pro-inflammatory cytokines and the generation of toxic free radicals, including nitric oxide (Greenberg et al. 1998).

Although a significant increase of iNOS is observed in macrophages in the brains of cattle and goats with natural listeriosis (J ungi et al. 1997), constitutive nNOS, which is one of the toxic mediators of ischemic brain injury, has not been well elucidated in bacterial meningoencephalitis. Therefore, the aim of this study was to examine the expression of both iNOS and nNOS in the brain in naturally occurring listeriosis. This study suggests that both nNOS and iNOS are constitutively expressed in brain cells, including neurons and some neuroglial cells, and that their expression increases in the brains of cattle with natural listeriosis in response to exogenous injury, such as bacterial infection. Therefore, nitric oxide produced by either nNOS or iNOS in neutrophils and macrophages may play an important role in eliminating L. monocytogenes and in the histopathology of brain tissue.

Selection of cases

\section{Materials and Methods}

Eight cattle of both sexes with natural listeriosis (1-to-5-year-old) and three controls (3-to-5-year-old) were studied. All the brains with natural listeriosis used in this study were from animals submitted for necropsy to the Pennsylvania Animal Diagnostic Laboratory System (PADLS) in Harrisburg, Pennsylvania. Animals with neurological paralysis, which had either been euthanized or found dead, were delivered to the necropsy laboratory. Three bovine brains were used as controls. The control animals had enteritis and no inflammation in the brain as determined by history, clinical signs, and by histological examination.

\section{Histopathological examination}

Specimens, including brainstem, cerebellum, and cerebrum, were routinely fixed in $10 \%$ buffered formalin, embedded in paraffin, sectioned at 4 microns, and stained with hematoxylin and eosin by routine histopathologic techniques. Brainstem sections of paraffin-embedded tissues from animals with characteristic histopathologic lesions of suppurative encephalitis were stored in the laboratory. In all the selected midbrains, L. monocytogenes was confirmed by immunohistochemistry using the antisera described below and used for the NOS immunohistochemistry. An ultrastructural study was excluded from this study because fresh tissues were not available and because immunohistochemical examination was sufficient to study NOS expression.

\section{Antisera and reagents}

The antisera used in this study were rabbit polyclonal antiserum against L. monocytogenes (Listeria $\mathrm{O}$ antiserum poly, serotypes 1 and 4, Difco, Detroit, MI), rabbit anti-glial fibrillary acidic protein (GFAP) (Sigma, St. Louis, MO), rabbit anti-iNOS (Sigma), rabbit anti-nNOS (Sigma), and rabbit anti-nitrotyrosine (Upstate Biotechnology Inc., Lake Placid, NY). Immunoperoxidase staining was done using the labeled [strept]avidin-biotin (LAB-SA) procedure (Zymed Laboratories, San Francisco, CA). Peroxidase-labeled isolectin B4 (Sigma) was used to detect macrophages.

\section{Immunohistochemistry}

Serial sections of midbrain, cerebrum, and cerebellum were deparaffinized and blocked with $3 \%$ hydrogen peroxide in distilled water for $10 \mathrm{~min}$. After washing with phosphate buffer, sections were blocked with normal goat serum for $10 \mathrm{~min}$. After blotting, the primary antisera were reacted for $60 \mathrm{~min}$, followed by biotinylated antisera (Zymed) for $15 \mathrm{~min}$ and [strept]avidin- biotin peroxidase (Zymed) for $15 \mathrm{~min}$. The staining was done using a LAB-SA Kit (Zymed) according to the manufacturer's instructions. The dilutions of primary antisera used in this study were as follows: anti-L. monocytogenes 1:1000, anti-GFAP 1:1000, anti-iNOS 1:100, anti-nNOS 1:100, and anti-nitrotyrosine 1:1000. To examine the specificity of each antiserum against either iNOS or nNOS, rabbit iNOS antiserum was reacted with nNOS peptide (Santa Cruz), or vice versa. For the negative control, primary antiserum was omitted or was replaced with normal rabbit serum (Zymed). For the detection of macrophages, peroxidase labeled isolectin B4 (1:10) was replaced with primary antiserum. Color development of peroxidase in NOS staining and lectin histochemistry was done using a diaminobenzidine substrate kit (Vector), while Listeria antigens were detected by AEC (Zymed). All incubations were at $36{ }^{\circ} \mathrm{C}$ using a Microprobe Staining System (Fisher Biotech, Fisher Scientific, St. Louis, MO). After finishing the immunoreaction, sections were counterstained with hematoxylin and mounted with Clearmount (Zymed). 


\section{Results}

Histopathology of bovine brains with natural listeriosis

Formalin-fixed brain tissues from animals with listeriosis showed typical suppurative meningoencephalitis in the midbrain (Plate VI, Fig. 1), occasionally in the cerebellum, and rarely in the cerebrum. The lesions consisted of microabscesses consisting of neutrophils, and perivascular cuffing of lymphocytes and macrophages. In some cases, axons near the inflammatory lesions were vesiculated. Inflammatory cells with pyknotic nuclei were commonly found within microabscesses. L. monocytogenes antigen was commonly found in microabscesses (Plate VI, Fig. 2) and the perivascular cuffs. Occasionally single, positivestaining bacteria were found in the neuronal processes.

Enhanced expression of nNOS and iNOS in L. monocytogenes-infected brain In control brains without encephalitis, nNOS was recognized in some neurons (Plate VII, Fig. 3, A), ependymal cells, and a few neuroglial cells. In brains with listeriosis, nNOS immunoreactivity was recognized in some neutrophils and macrophages in microabscesses, in areas of perivascular cuffing (Fig. 3, C and D), and in some GFAP-positive astrocytes distant from perivascular cuffs (Fig. 3, E and F). Astrocytes were identified by GFAP labeling and macrophages were identified in adjacent sections by isolectin B4 labeling. Immunostaining in the inflammatory cells was more intense in a severe case than in a mild case. This suggests that nNOS is an enzyme that generates nitric oxide in encephalitis due to L. monocytogenes.

The immunoreactivity of iNOS in control brains was seen in occasional neurons (Plate VII, Fig. 4, A), some neuroglial cells, and choroid plexus cells. iNOS immunostaining was present in macrophages (Fig. 4, C and D) and some astrocytes in the brain stem of affected animals (Fig. 4, E and F), but with less intensity than nNOS. The iNOS-immunoreactivity in inflammatory cells was somewhat similar to that of nNOS, in that iNOS was recognized mainly in activated macrophages in areas of perivascular cuffing and in microabscesses. However, iNOS-immunoreactivity was not observed in neutrophils in either perivascular cuffs or in microabscesses. These observations indicate that the staining patterns of the two molecules differ with the cell type involved.

To determine the capacity for nitric oxide generation, immunostaining of nitrotyrosine was carried out. In a few lesions, both iNOS- and nNOS-positive cells (mainly macrophages) were also positive for nitrotyrosine, an end-product of NOS (data not shown).

\section{Discussion}

The involvement of iNOS in bovine listeriosis was reported previously; increased expression of iNOS and resultant NO generation were confirmed in brains with listeriosis and in cultured macrophages from the Listeria-affected cattle (Jungi et al. 1997). These findings suggest that increased expression of iNOS in macrophages is associated with antibacterial effects and injury of the affected brain. Although our findings were consistent with those previously reported as a regards iNOS expression in macrophages, iNOS immunostaining was also recognized in neurons and astrocytes in the brains of cows with listeriosis, similar to the iNOS immunostaining seen in cultured stimulated neurons (Heneka et al. 1998). In addition, multiple factors may have produced this minor discrepancy, including the different (1) detection system (Zymed Histoplus immunostaining kit) and (2) source of primary antisera (rabbit anti-inducible NOS from Sigma) used in this study. The previous study used the ABC staining system (Immunodiffusion, Geneva, Switzerland) and rabbit polyclonal anti-mouse iNOS antisera (Upstate Biotechnology Inc., Lake Placid, NY). 
nNOS is an inflammatory mediator in arthritis ( $\mathrm{Wu}$ et al. 1999), as well as a neurotransmitter. The important finding in this study was that nNOS is not only expressed in some neurons, but also in hematogenous macrophages and neuroglial cells including astrocytes. This finding is further supported by the detection of nNOS mRNA in hematogenous cells, including macrophages, T cells (Reiling et al. 1994), and neutrophils in vitro (Greenberg et al. 1998). These results suggest that constitutive nNOS from brain cells becomes a source of NO in pathologic brain tissues, although not all NOS isozymes are associated with the generation of nitric oxide. However, we confirmed that some cells, including macrophages, expressed both iNOS and nNOS, and these cells were also positive for nitrotyrosine. This suggests that iNOS or nNOS are involved in the generation of nitric oxide, although it is unclear which isoform of NOS is directly associated with nitrotyrosine formation in Listeria encephalitis.

The functional roles of iNOS and nNOS in generating nitric oxide remain to be studied in CNS inflammation. Although many researchers have studied the detrimental effects of nNOS in CNS trauma (Sharma et al. 1996) and hypoxic injury (Black et al. 1995), nNOS is also very important for host recovery against viral infection in mice (Komatsu et al. 1999) and peripheral nerve regeneration (Gonzalez-Hernandez and Rustioni 1999). In addition, NO produced by iNOS may be either beneficial by promoting the differentiation and survival of neurons (Munoz-Fernandez and Fresno 1998), or detrimental by inducing neuronal cell death (Zhou et al. 1999). Taking all into consideration, we postulate that NO has a dual effect, beneficial or harmful, on host cells, depending on the stimulation conditions (Willenborg et al. 1999).

In summary, these results suggest that both nNOS and iNOS are constitutively expressed in brain cells, including neurons and some neuroglial cells, and that in the brains of cattle with natural listeriosis their expression increases in response to exogenous injury, such as bacterial infection. In acute, natural bovine listeriosis, nitric oxide produced by either nNOS or iNOS in neutrophils and macrophages may play an important role in eliminating L. monocytogenes and in the histopathology of brain tissue. Since natural listeriosis causes sudden death in cattle, the functional role of nitric oxide, via either iNOS or nNOS, needs further study.

\section{Acknowledgments}

The authors greatly appreciate Carol Robinson's technical support. The authors also thank Dr. T. W. Jungi, Institute of Veterinary Virology and Animal Pathology, University of Bern, Switzerland for critical discussion of this manuscript. This work was supported by a grant from the Chongbong Academic Research Fund of the Cheju National University Development Foundation.

\section{References}

BLACK, S. M., BEDOLLI, M. A., MARTINEZ, S., BRISTOW, J. D., FERRIERO, D. M., SOIFER, S. J. 1995 : Expression of neuronal nitric oxide synthase corresponds to regions of selective vulnerability to hypoxiaischaemia in the developing rat brain. Neurobiol. 2: 145-155

BREDT, D. S., SNYDER, S. H. 1992: Nitric oxide, a novel neuronal messenger. Neuron. 8: 3-11

COOPER, J., WALKER, R. D. 1998: Listeriosis. Vet. Clin. North. Am. Food Anim. Pract. 14: 113-125

CROSS, A. H., MANNING, P. T., STERN, M. K., MISKO, T. P. 1997: Evidence for the production of peroxynitrite in inflammatory CNS demyelination. J. Neuroimmunol. 80: 121-130

DRAMSI, S., LEVI, S., TRILLER, A., COSSART, P. 1998: Entry of Listeria monocytogenes into neurons occurs by cell-to-cell spread: an in vitro study. Infect. Immun. 66: 4461-4468

GONZALEZ-HERNANDEZ, T., RUSTIONI, A. 1999: Expression of three forms of nitric oxide synthase in peripheral nerve regeneration. J. Neurosci. Res. 55: 198-207

GREENBERG, S. S., OUYANG, J., ZHAO, X., GILES, T. D. 1998: Human and rat neutrophils constitutively express neuronal nitric oxide synthase mRNA. Nitric Oxide 2: 203-212

HENEKA, M. T., LOSCHMANN, P. A., GLEICHMANN, M., WELLER, M., SCHULTZ, J. B., WULLNER, U. KLOCKGETHER, T. 1998: Induction of nitric oxide synthase and nitric oxide-mediated apoptosis in neuronal PC12 cells after stimulation with tumor necrosis factor-alpha/lipopolysaccharide. J. Neurochem. 71: 88-92

HOOPER, D. C., OHNISHI, S. T., KEAN, R., NUMAGAMI, Y., DIETZSCHOLD, B., KOPROWSKI, H. 1995: 
Local nitric oxide production in viral and autoimmune disease of the central nervous system. Proc. Natl. Acad. Sci. U.S.A. 92: 5312-5316

IWAHASHI, T., INOUE, A., KOH, C. S., SHIN, T. K., KIM, B. S. 1999: Expression and potential role of inducible nitric oxide synthase in the central nervous system of Theiler's murine encephalomyelitis virus-induced demyelinating disease. Cell Immunol. 194: 186-193

JOHNSON, G. C., FALES, W. H., MADDOX, C. W., RAMOS-VARA, J. A. 1995: Evaluation of laboratory test for confirming the diagnosis of encephalitic listeriosis in ruminants. J. Vet. Diagn. Invest. 7: 223-228

JUNGI, T. W., PFISTER, H., SAGER, H., FATZER, R., VANDEVELDE, M., ZURBRIGGEN, A. 1997 : Comparison of inducible nitric oxide synthase expression in the brain of Listeria monocytogenes-infected cattle, sheep and goats and in macrophages stimulated in vitro. Infect. Immun. 65: 5279-5288

KOMATSU, T., IRELAND, D. D., CHEN, N., REISS, C. S. 1999: Neuronal expression of NOS-1 is required for host recovery from viral encephalitis. Virology 258: 389-395

KRUEGER, N., LOW, C., DONACHIE, W. 1995: Phenotypic characterization of the cells of the inflammatory response in ovine encephalitic listeriosis. J. Comp. Pathol. 113: 263-275

MONCADA, S., PALMER, R. M. J., HIGGS, E. A. 1991: Nitric oxide: physiology, pathophysiology, pharmacology. Pharmacol. Rev. 43: 109-134

MUNOZ-FERNANDEZ, M. A., FRESNO, M. 1998: The role of tumour necrosis factor, interleukin 6, interferongamma and inducible nitric oxide synthase in the development and pathology of the nervous system. Prog. Neurobiol. 56: 307-340

MURPHY, S., SIMMONS, M. L., AGUllo, L., CARCIA, A., FEINSTEIN, D.L., GALEA, E., REIS, D. J., MINC-GOLOMB, D., SCHWARTZ, J. P. 1993: Synthesis of nitric oxide in CNS glial cells. T.I.N.S. 16: $323-$ 328

OTTER, A., BLACKMORE, W. F. 1989: Observation on the presence of Listeria monocytogenes in axons. Acta Microbiol. Hung. 36: 125-131

REILING, N., ULMER, A. J., DUCHROW, M., ERNST, M., FLAD, H. D., HAUSCHILDT, S. 1994: Nitric oxide synthase: mRNA expression of different isoforms in human monocytes/macrophages. Eur. J. Immunol. 24: 1941-1944

SHARMA, H. S., WESTMAN, J., OLSSON, Y., ALM, P. 1996: Involvement of nitric oxide in acute spinal cord injury: an immunocytochemical study using light and electron microscopy in the rat. Neurosci. Res. 24:373-384

VAN DAM, A. M., BAUER, J., MAN-A-HING, W. K., MARQUETTE, C., TILDERS, F. J., BERKENBOSCH, F. 1995: Appearance of inducible nitric oxide synthase in the rat central nervous system after rabies virus infection and during experimental allergic encephalomyelitis but not after peripheral administration of endotoxin. J. Neurosci. Res. 40: 251-260

WILLENBORG, D.O., STAYKOVA, M.A., COWDEN, W.B. 1999: Our shifting understanding of the role of nitric oxide in autoimmune encephalomyelitis: a review. J. Neuroimmunol. 100: 21-35.

WU, J., LIN, Q., LU, Y., WILLIS, W. D., WESTLUND, K. N. 1998: Changes in nitric oxide synthase isoforms in the spinal cord of rat following induction of chronic arthritis. Exp. Brain Res. 118: 457-465

ZHOU, Y., ZHAO, Y. N., YANG, E. B., LING, E. A., WANG, Y., HASSOUNA, M. M., MACK, P. 1999: Induction of neuronal and inducible nitric oxide synthase in the motoneurons of spinal cord following transient abdominal aorta occlusion in rats. J. Surg. Res. 87:185-193 
Plate VI

Shin T. et al.: Neuronal... pp. 43-47
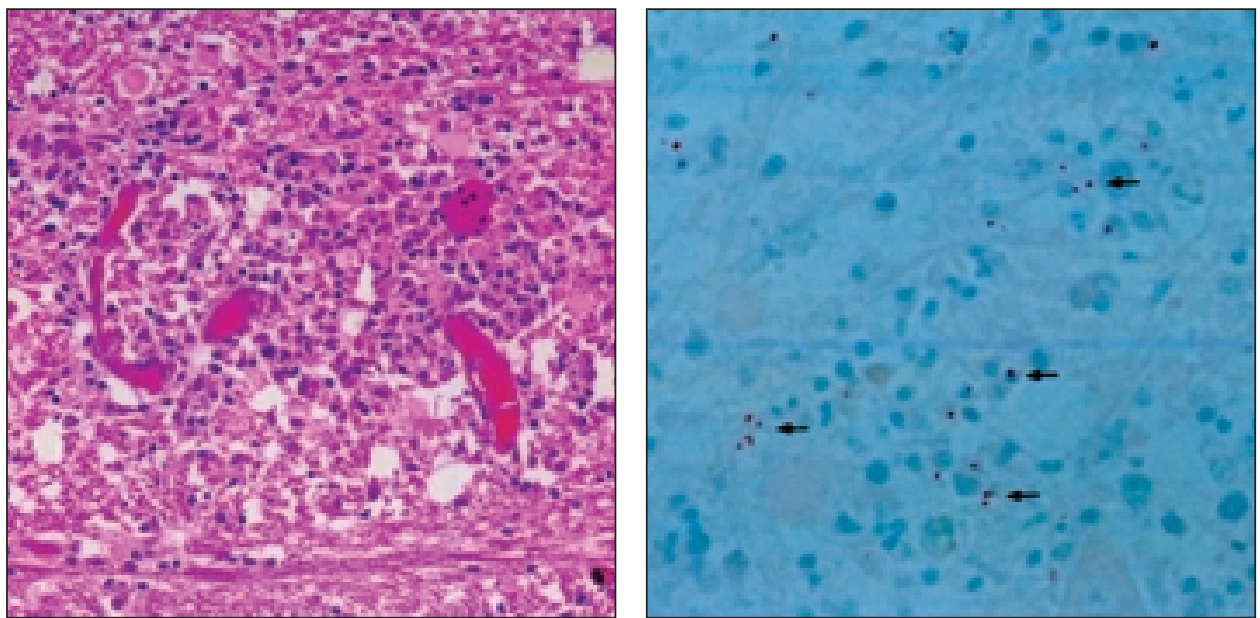

Plate VI, Fig. 1. (left) Brain of a cow with listeriosis, showing meningoencephalitis. H-E, magnification $\times 150$. Fig. 2. (right) Detection of L. monocytogenes in the bovine brain. A small number of rod-shaped bacteria with immunoreactivity to anti-L. monocytogenes antibody are observed in an inflammatory microabscess. AEC was used for color development. Counterstained with hematoxylin, $\times 300$.

Plate VII, Fig. 3. Immunostaining of neuronal NOS in brain sections of control cattle (A) and cattle with natural listeriosis (C and $\mathrm{E})$. nNOS immunoreactivity is seen in some neurons (A) in the brain of a control animal with a non-neurological disease, but not in a negative control (B). In listeric encephalitis (C-F), nNOS was present in the inflammatory cells $(C)$ in microabscesses in which some cells were stained with isolectin B4 (D), suggesting that macrophages express nNOS. In a lesion distant from perivascular cuffing ( $\mathrm{E}$ and $\mathrm{F}$ ), nNOS immunoreactivity (E) was co-localized in GFAP-positive cells (F) in an adjacent section. Arrows indicate the same cell group in adjacent sections (C and D, E and F). DAB was used for color development. Counterstained with hematoxylin, $\times 300$.

Plate VIII, Fig. 4. Immunohistochemical staining of inducible NOS in bovine brain in controls (A) and natural listeriosis $(\mathrm{C}-\mathrm{F})$. iNOS immunoreactivity $(\mathrm{C}$ and $\mathrm{E})$ was recognized in isolectin B4-labeled macrophages (D) and GFAP-positive astrocytes (F), respectively. Negative control without primary antisera showed no staining (B). Arrows indicate the same cell group in adjacent sections ( $\mathrm{C}$ and D, E and F). DAB was used for color development. Counterstained with hematoxylin, $\times 300$. 


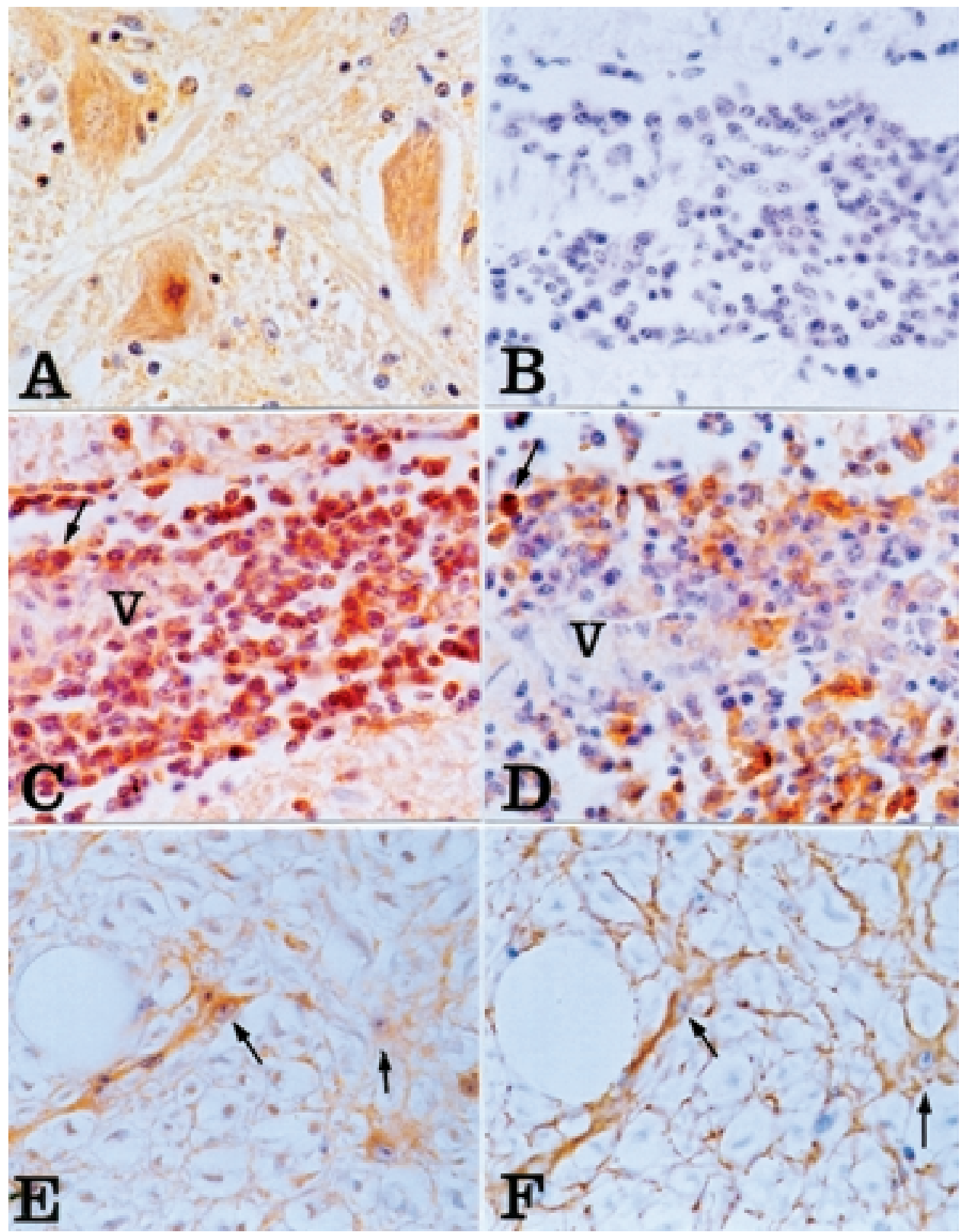


Plate VIII.

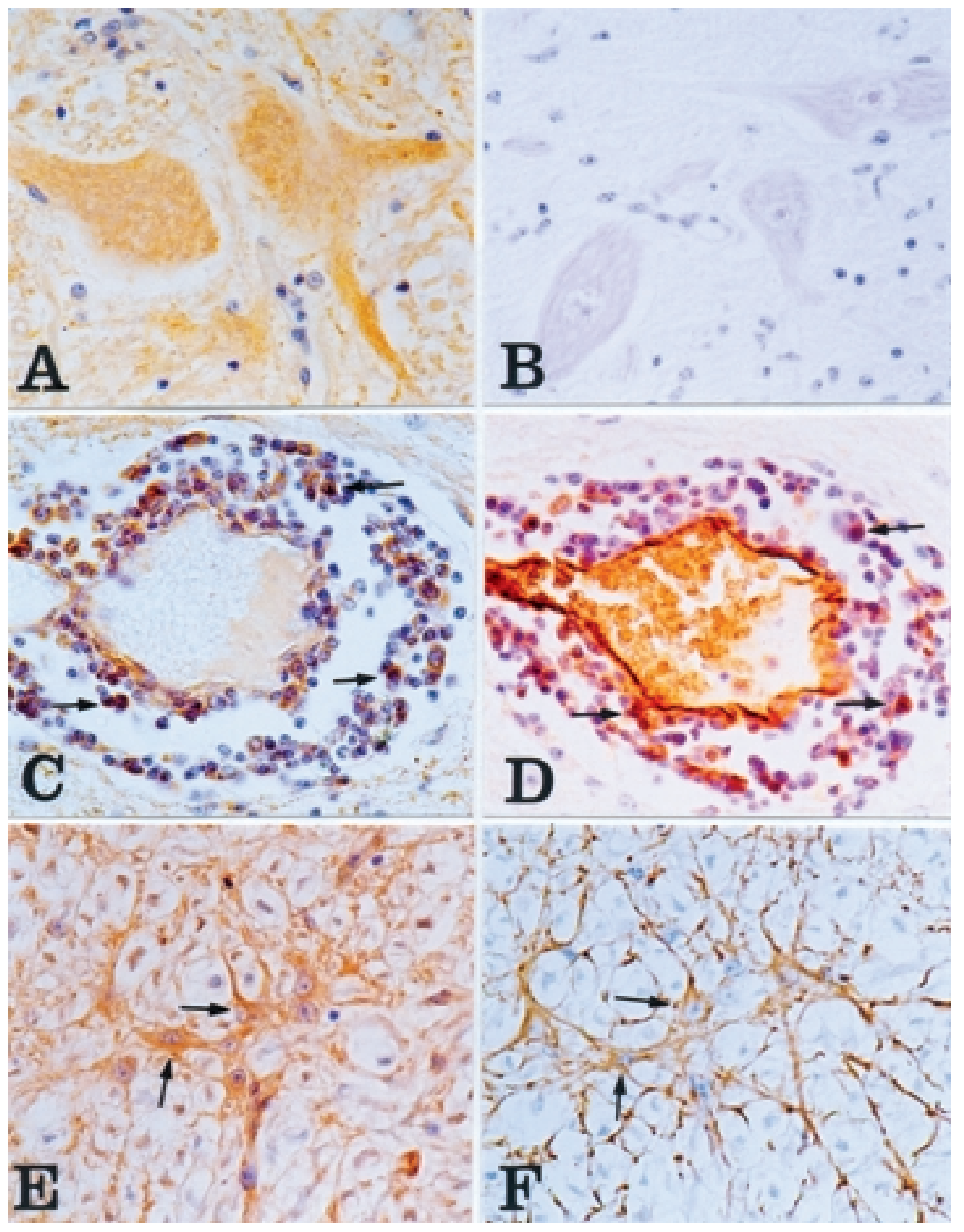

